\title{
Detection of In-site and Cause Analysis of Splitting Cracks of Vertical Wall for Large-scale three-direction Pre-stressed Aqueduct
}

\author{
T. HUANG, H.F. LI, G.X. ZHANG, L.N. ZHAO, F. SHANG, X.M. ZHU, D.L. LI, \\ S.K. FENG
}

State Key Laboratory of Simulation and Regulation of Water Cycle in River Basin, China Institute of Water Resources and Hydropower Research, Beijing, China

Rongwu Expressway of Penglai Management Office, Yantai, ShanDong Province, China

School of Naval Architectural, Ocean and Civil Engineering, Shanghai Jiao-tong University, Shanghai, China

KEYWORDS: Splitting Cracks of Vertical Wall; Large Scale Three-direction Pre-stressed Aqueduct; Non-destructive Test of Concrete; Crack Detection; Cause Analysis

ABSTRACT: Splitting cracks were found in the three-directional pre-stressed aqueduct of a large scale diversion project in north China. Based on internal structural characteristics of the vertical wall of aqueduct, the Impact Imaging Method (IIM) was adopted for the detections of cracks distribution, seriousness and damage area ratio of the internal cracks and the damage cause analysis. The core test and numerical simulation by 3D-FEM with prefabricated joints element were also adopted for verifications. The drilled cores showed that the IIM has high accuracy and resolutions on the internal crack distributions. It provides reliable fundamental data for decisions of the wall repair strategies. Both field test results and numerical analysis showed that the frost of the water inside the corrugated pipes that contained the pre-stress tendon led to the internal splitting cracks, which was due to the incompact grouting into the pipes. This accident teaches us the significance for the grouting quality control of the corrugated pipes inside the post-tensioned structures in cold regions of northern China.

\section{Introduction}

In recent years, China has made significant progress in strengthening its national economy. Concrete structures and buildings, including aqueducts, bridges, tunnels, and underground powerhouses are becoming larger, and in some cases, their construction has been accompanied by engineering accidents. Thus, inspecting engineering quality is extremely important in ensuring the quality of projects. Most large facilities adopt pre-stressed concrete structures. The complicated manufacturing process, difficult quality control, and high density of reinforcement in the structure are challenge faced in quality inspection. At present, concrete nondestructive testing methods include rebound, ultrasonic, and geological radar methods (CWIS 2005). However, the rebound method can only detect the surface strength of the structure, but not the internal state of a structure with thick concrete. The ultrasonic method can detect the internal state of concrete in principle. However, the detection result is extremely susceptible to the influence of the water and steel in concrete. The geological radar method is also affected by the distribution of reinforcement(Che 2011,Wan 2012\&Deng 2008). Thus, developing new non-destructive detection methods is crucial(Liu 2013\&Huang 2015).In view of the aforementioned findings, this paper proposes and 
tests a method called impact imaging (a type of nondestructive detection method) to detect the internal cracks in concrete. The detection result demonstrates that this method can effectively detect the internal cracks in concrete and is characterized by high speed and high efficiency. The approach is also unaffected by internal reinforcement.

\section{Survey of detection project}

Large-scale pre-stressed concrete walls of aqueduct are an important component of a water diversion facility in North China. The main body of this project consists of four three-direction pre-stressed concrete walls with a length of $640 \mathrm{~m}$. Each wall is divided into 16 crosses that are 40.0 $\mathrm{m}$ long, $9.1 \mathrm{~m}$ high, and $0.7 \mathrm{~m}$ thick each. The total investment in the project is more than 200 million yuan. The facility was constructed in June 2010. In October 2012, cracks were observed on the surface of the wall sections casted the previous year. A hollow sound was heard upon knocking on the structure. Thus, the wall was suspected to be non-dense.

The large-scale water conservancy facility has large design flow, deep water level, and high elevation. The design flow is $230 \mathrm{~m} 3 / \mathrm{s}$ and $250 \mathrm{~m} 3 / \mathrm{s}$ at most. The maximum velocity of flow is 1.7 $\mathrm{m} / \mathrm{s}$. The highest water head is $7.1 \mathrm{~m}$. The net height from the ground to the facility is approximately $14 \mathrm{~m}$. The volume of the facility is roughly $105 \mathrm{~m} 3$. If a collapse accident would occur in this facility, the consequences would be catastrophic.

The wall reinforcement is shown in Fig. 1. A $\varphi 32$ finishing threaded steel is used as the main reinforcement with an interval of $25 \mathrm{~cm}$. A $\varphi 16$ finishing threaded steel is adopted as the auxiliary

reinforcement with an interval of $15 \mathrm{~cm}$. PSB785 $\mathrm{MPa} \Phi^{\mathrm{ps}} 32 \mathrm{~mm}$ finishing threaded steel is used as

the vertical pre-stressed reinforcement. Except for the reinforcement in Fig.1, five 9-7 $\Phi^{\mathrm{s}} 15.2$ pre-stressed strands in Class $1860 \mathrm{MPa}$ are equipped. These reinforcements and strands are widely distributed in the walls, forming the interference to electromagnetic wave and ultrasonic wave detection. Geological radar and ultrasonic inspection as well as other traditional methods cannot be employed for inspection. Thus, impact imaging method is used for inspection based on a detailed examination of this project.

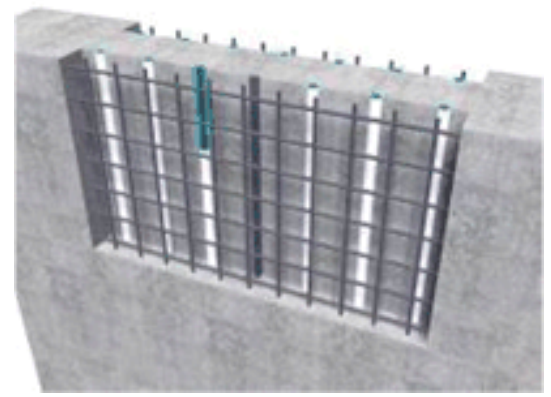

Fig. 1 Reinforced and pre-stressed reinforcement configuration in a vertical wall

\section{In-site detection}

Detection method

As shown in Fig.2, V1, V2, V3 represents the elastic wave velocity of media 1, media 2, media 3 respectively, Media 2 can be homogenous of half space. If $\mathrm{V} 2=\mathrm{V} 3$, the media of homogenous of 
half space has no defect; if $\mathrm{V} 2 \neq \mathrm{V} 3$, the media 3 can be regarded as a local defect; if $\mathrm{V} 3=0$ approximately, media 3 can be considered as a void under a concrete specimen. As shown in Fig.3, when wave incidents from media 1 into the interface with media 2, it will be reflected, refracted and converted. Take $\mathrm{P}$ wave as an example, the direction of wave propagation complies with Snell's law as bellow:

$V_{p 1} / \sin \left(\alpha_{1}\right)=V_{p 1} / \sin \left(\alpha_{2}\right)=V_{p 2} / \sin \left(\alpha_{3}\right)$

$=V_{s 1} / \sin \left(\beta_{1}\right)=V_{s 2} / \sin \left(\beta_{2}\right)$

Where $V_{p 1}, V_{p 2}$ are the velocity of $P$ wave of media 1 and media 2 respectively, $V_{s 1}$ and $V_{s 2}$ are the velocity of $S$ wave of media 1 and media $2, \alpha_{1}, \alpha_{2}$ and $\alpha_{3}$ are the incident angle ,reflection angle and refraction angle, $\beta_{1}$ and $\beta_{2}$ are the reflection angle and refraction angle of converted SV wave.

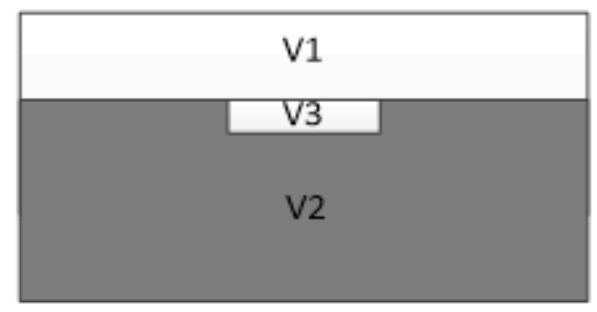

Fig.2 Behaviors of incident wave on the interface

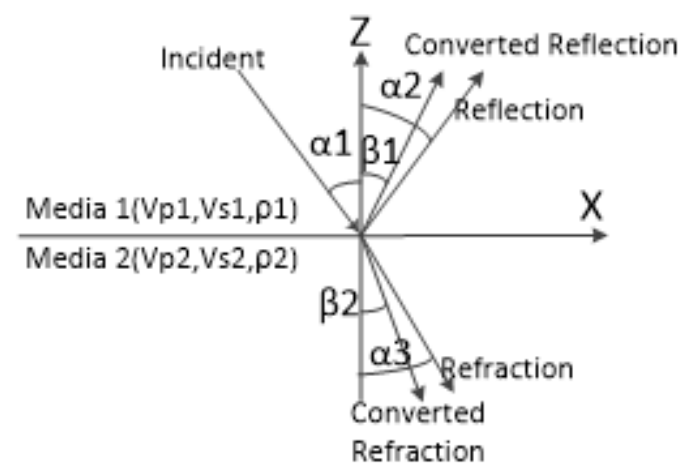

Fig.3 Behaviors of incident wave on the interface

The coefficients of reflection and refraction, i.e. the amplitude ratio between incident wave and reflection wave as well as refraction wave are very important for seismic exploration. Though they can be calculated by solving wave equation with boundary conditions, the expressions are complicated. However, if the wave incidents normally to the interface $\left({ }^{\alpha_{1}}=0\right)$, there will be no wave conversion from $\mathrm{P}$ to $\mathrm{S}$ as well as from $\mathrm{S}$ to $\mathrm{P}$, the expressions of reflection coefficients become simples as :

$$
\begin{array}{r}
R_{p}=\frac{\rho_{2} V_{p 2}-\rho_{1} V_{p 1}}{\rho_{2} V_{p 2}+\rho_{1} V_{p 1}} \\
R_{s}=\frac{\rho_{2} V_{s 2}-\rho_{1} V_{s 1}}{\rho_{2} V_{s 2}+\rho_{1} V_{s 1}}
\end{array}
$$


Where $\mathrm{Rp}$ is the reflection coefficient of $\mathrm{P}$ wave and $\mathrm{Rs}$ is reflection coefficient of $\mathrm{S}$ wave.

Based on the elastic wave propagation theory, as shown in Fig.4, the seismic wave is generated by mechanical impact on the surface of the concrete specimens pouring from two materials. Let's consider an observation point in the vicinity of the source point. If the offset of observation point from the source is short enough in respect to the thickness of the concrete specimens, the seismic wave can be handled as normally to incident on the interface between media 1 and media 2 , and the reflection coefficient can be calculated using eq.(2) or eq.(3). The seismic wave observed at the observation point can be expressed as:

$$
\begin{aligned}
& A_{1}=A_{0} R_{1} e^{i w} \\
& A_{2}=A_{1} R_{0} R_{1} e^{i w}=A_{0} R_{1}^{2} R_{0} e^{i 2 w} \\
& A_{3}=A_{2} R_{0} R_{1} e^{i w}=A_{0} R_{1}^{3} R_{0}^{2} e^{i 3 w} \\
& \quad \ldots \ldots \ldots . \\
& A_{n}=A_{n-1} R_{0} R_{1} e^{i w}=A_{0} R_{1}{ }^{n} R_{0}^{n \cdot 1} e^{i n w}
\end{aligned}
$$

Where R0 is the reflection coefficient of the free surface and R1 is the reflection coefficient of interface 1. Due to the density of the air above the concrete specimens is very small, so R0 $\approx-1$, the strength of the wave observed depends on the reflection coefficient of interface 1 . The eiw is phase difference of wave fluctuations caused by the first spread between interfaces, A0 is the initial amplitude of the incident wave, and so on.

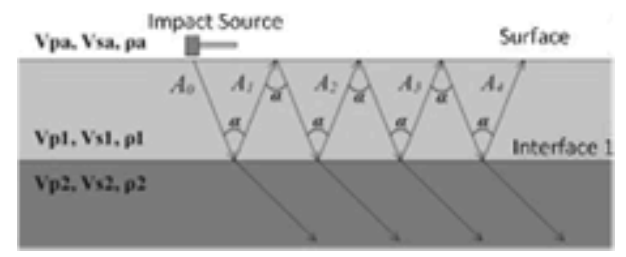

Fig.4 Multiples reflection of elastic waves between the interface

According to elastic wave theory, when the impact is exerted on the surface of the medium, the elastic wave is generated in the medium. The field distribution of the elastic wave is closely related to the internal structure of the medium. Fig.5 shows that when the impact load is exerted on the surface of the medium, the detector is used to receive and record the elastic wave at D distant to the impact point. Distance D (called as offset distance) and the impact strength are kept unchanged. The impact-receiving system is moved to the next detection point to re-excite and receive the elastic wave. If the internal structure of the medium is unchanged, the received elastic waveform is also unchanged. By contrast, if the internal structure of the medium is changed, e.g., if cracks or holes appear, the received waveform is changed. Thus, the change in the characteristics of the waveform can be a basis to assess if the medium has a defect, as shown in Fig. 6. 


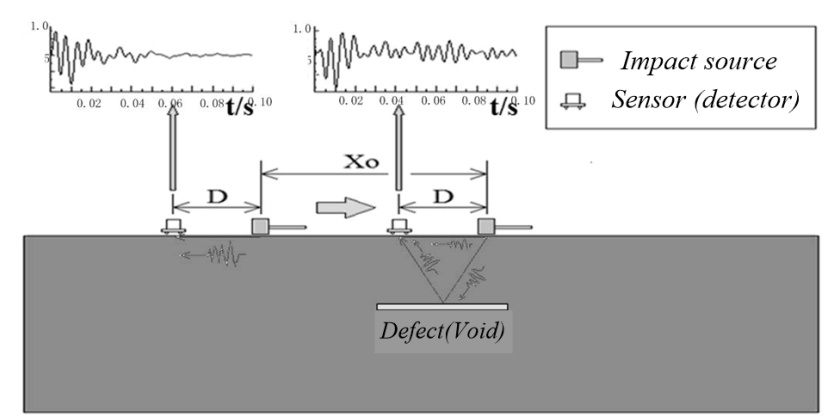

Fig. 5 Data collection of impact image method

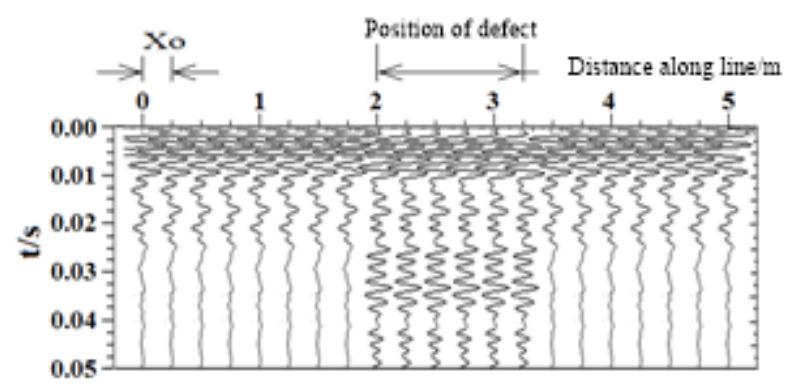

Fig. 6 Response wave of internal structure

\section{Detection instrument}

The detection system consists of the detector (probe), data acquisition instrument, impact source, cable, power supply, and notebook computer. The special probe is a moving coil velocity sensor with a natural frequency of $100 \mathrm{~Hz}$. The data acquisition instrument is 24bit $\Sigma-\Delta$ type high-precision broadband seismic instrument produced by US Geomatrics. The impact source is a steel hammer with mass of $250 \mathrm{~g}$. The field notebook computer is used to control and record the data. The power supply is a universal 12-V vehicle battery, as shown in Fig. 7.

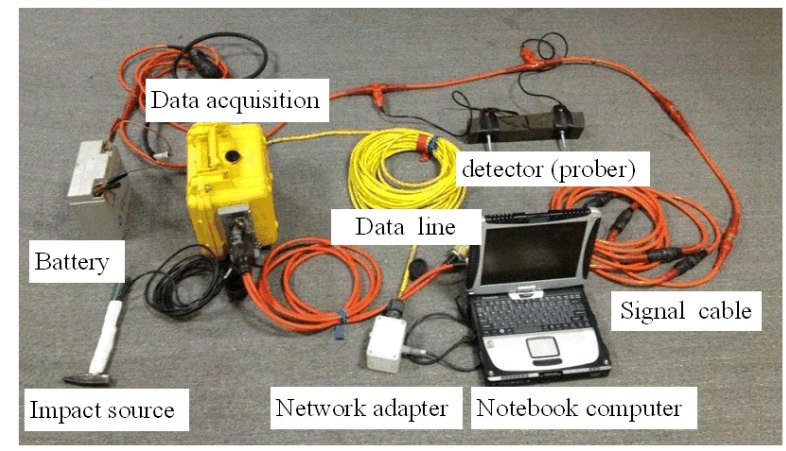

Fig. 7 Detection system configuration

In the detection system, the impact hammer is used to excite the elastic wave. The elastic wave is released from the impact source. A part of the wave spreads to the detector along the surface, whereas another part of the wave extends to the interior of the medium and the detector through reflection. The signal received by the detector is transmitted to the data acquisition instrument through the cable and the computer to be digitally recorded through the network adapter and LAN interface after amplification, filtering, and model or digit conversion. 


\section{Line layout and data acquisition}

Line layout

Based on the functions of the wall structure and water conservancy facilities, seven lines were laid on each side of wall to be detected, called L0-L6 (see Fig. 8) from low to high. The spacing of the wall bottom line was $0.3-0.5 \mathrm{~m}$, and the line spacing of other parts was $1.0 \mathrm{~m}$. The top of the wing wall is set as the zero point of height. Thus, lines L0-L6 were 0.3, 0.6, 1.1, 1.6, 2.6, 3.6, and $4.6 \mathrm{~m}$ high, respectively.

All the lines were $40 \mathrm{~m}$ long. In each line, the detection points were distributed with a spacing of $0.2 \mathrm{~m}$. The whole detection covered five walls. The total detection area was $40 \mathrm{~m}(\mathrm{~L}) \times 7.1 \mathrm{~m}(\mathrm{~W}) \times$ 5 (number of walls $) \times 2($ side $) \times 5($ section $)=14200 \mathrm{~m} 2$. The total number of detection points was $54,600$.

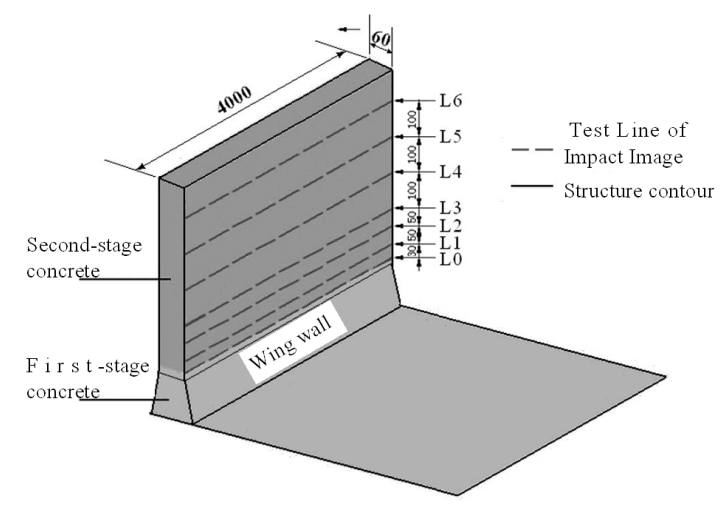

Fig. 8 Schematic of measured line position $(\mathrm{cm})$

Data acquisition

Before data acquisition, lines were first drawn on the walls according to the design of the line. The detection points in each line were marked with a spacing of $0.2 \mathrm{~m}$. The set detection point density should not only ensure the optimal detection result but also consider the efficiency of data acquisition. The parameter of the impact imaging method is mainly the source offset. The impact imaging method primarily utilizes the near-source elastic wave. Thus, the source offset is generally less than or equal to the depth of the expected defect. Given that the maximum depth of the crack in the wall reached 0.2-0.3 m, and with large detection workload and accuracy, the impact source offset of this detection was set to $0.2 \mathrm{~m}$ and the density of the detection points (spacing) was also $0.2 \mathrm{~m}$.

During detection, a person held the special detection prober and aligned the first detector to the first detection point and the second detector to the second detection point. The detector was compacted vertically to the wall. A $200 \mathrm{~g}$ steel hammer was used to knock the third detection point, and the data were recorded. After completing the detection at one point, the entire system was moved $0.2 \mathrm{~m}$ forward. The process was repeated until the detection in one line was completed.

Fig.9 illustrates the elastic waveform recorded by the instrument (called the impact response waveform). The figure indicates that the collected data are classified into two waveforms: one waveform is higher in amplitude and is long-lasting, whereas the other waveform is lower in amplitude and is short-lasting. Some higher amplitude waveforms that are aligned to the position generate a dull sound, and exciting the elastic wave produces a hollow sound. However, the lower amplitude waveforms aligned to the position generate a brisk sound, indicating the high density of concrete. 


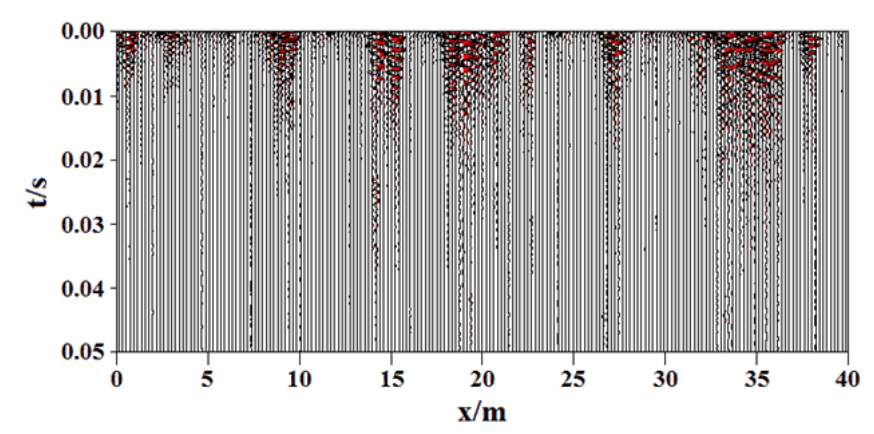

Fig. 9 Wave of impact response

\section{Data analysis}

Data analysis process

Analyzing the impact image can be divided into three parts, namely, data processing, numerical stimulation, and result interpretation.

Data processing aims to edit, filter, and mathematically transform the detection data to suppress the noise in the data and represent the useful information in special form. Data processing mainly includes data pre-processing, data normalization, waveform processing, wave field separation, and calculation of impact response.

Numerical simulation is conducted to establish the mathematical physics model of the structure to be detected according to the internal structure of the detected object. The principle and parameters of detection are used to establish the mathematical model of detection. Finally, the numerical simulation is used to solve the wave equation based on the given model and to theoretically analyze the relation between crack and impact response intensity. The results provide a basis for the actual detection data analysis and result interpretation.

The engineering interpretation of the result of the numerical simulation result involves defining the impact response intensity distribution map, which is the information required in engineering, namely, non-dense situation in concrete wall, and calculating the percentage of the hollow region.

Setting of defect judgment index

In summary, the engineering interpretation of the analysis result mainly depends on the result of the numerical simulation. The minute adjustment of the judgment criteria is based on the drilling verification.

The model used in the numerical simulation is presented in Fig. 10. To minimize the boundary effect of the numerical simulation, the model is set to $20.0 \mathrm{~m}$ in length and $0.7 \mathrm{~m}$ in thickness. One $0.01 \mathrm{~m}$-wide, $2.0 \mathrm{~m}$-long, and $0.1-\mathrm{m}$ deep crack is set at the center of the model. The velocity of the longitudinal wave of dense concrete is $5000 \mathrm{~m} / \mathrm{s}$, and the velocity of the cracked concrete is 3000 $\mathrm{m} / \mathrm{s}$. The offset distance is set to $0.2 \mathrm{~m}$, and the moving distance is $0.2 \mathrm{~m}$. The center frequency is set to $5000 \mathrm{~Hz}$. The physical parameters of the model are estimated based on the data of the surface wave detection of the actual wall. Other parameters are consistent with the actual detection values. The numerical simulation is conducted using finite element wave equation.

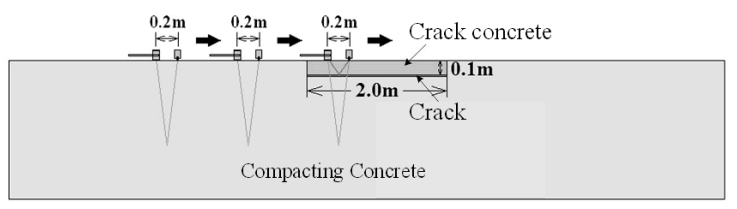

Fig.10 Numerical simulation model 


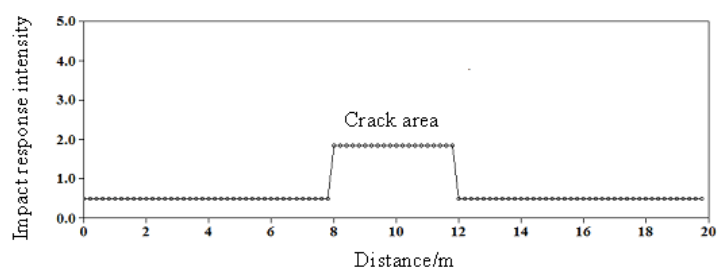

Fig. 11 Impact response intensity of numerical simulation (dimensionless)

Fig.11 shows the impact response intensity calculated based on the impact response wave (average amplitude P), which indicates the energy of the response wave. The figure shows that in the dense area, $\mathrm{P} \leq 0.5$, and in the cracked area, $\mathrm{P} \geq 2.0$. The difference between the two areas is 4 times and higher, and is therefore significant.

Based on the result of the theoretical calculation, as well as the core-taking verification result, and considering the effect of the boundary of the cracked areas, the defect judgment criterion is finally determined as follows: $\mathrm{P} \leq 1.0$, dense; $\mathrm{P}=1.0-1.75$, unclearly judged; and $\mathrm{P} \geq 1.75$, hollow.

\section{Data analysis results}

Data analysis results

The results of the data analysis are shown in Figs. 12a, 12b. Fig. 12a illustrates the plane distribution graph of a section of the wall crack. The graph is generated by calculating the impact response intensity of each detection point and drawing the configuration graph of the impact response intensity. Finally, the established engineering interpretation criterion is used to transform the impact response intensity configuration graph to the crack configuration graph. The figure indicates that cracks or hollows are present in the center of walls and a small unclear area at the edge of crack areas exist. This area is part of the crack tip area. Some cracks are closed. Thus, their performance is unclear in the impact response waveform.

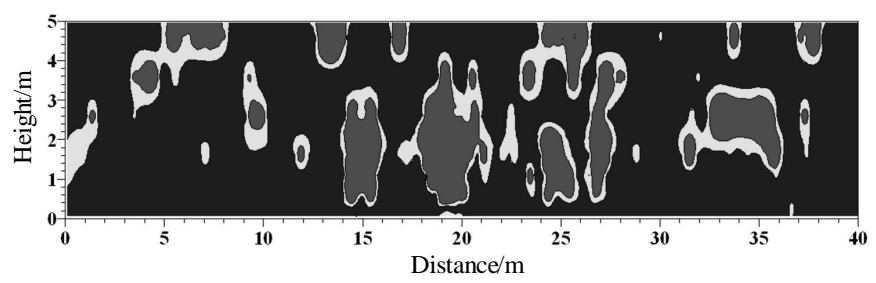

Fig. 12a Internal crack configuration on wall
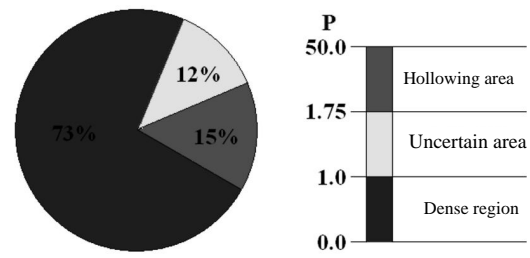

Fig. 12b Percentage of internal cracks on vertical wall

Fig.12b shows the statistical data of the density of the wall. P stands for intensity of impact response. The figure indicates that the dense part of the wall accounts for $73 \%$, the hollow area (crack) accounts for $15 \%$, and the region that is difficult to assess accounts for $12 \%$.

Core-taking verification results

Core-taking verification is adopted to confirm the accuracy of the results. A total of 59 core-taking points are arranged according to the detection result. Of the 59 core-taking points, 46 points are 
arranged in the hollow area verified by the detection result, 11 points are arranged in the dense area, and 2 are arranged in the unclear area. Of the 46 core-taking points in the hollow area, 44 cores were successfully obtained. Two obtained cores were stopped because of the reinforcement in drilling. A total of 46 core samples in the hollow area were confirmed to have hollow cracks. Of the 11 core-taking points in the dense area, 11 cores were successfully obtained without drilling cracks. Of the two core-taking points arranged in the unclear area, two core samples with cracks were successfully obtained.

\section{Analysis of cause of crack}

Analysis of cause of crack

Theoretically, many factors cause internal cracks in a concrete wall. No problem was encountered on the cast wall before the first winter because of long-term maintenance. After the winter, cracks were observed. Results of the detection revealed that water existed in the corrugated pipe and formed a jet in the borehole drainage. Thus, the corrugated pipe grouting was assumed to be not full and the frost heave of the internal water caused the cracks. The corrugated pipe is made of plastic and cannot be destroyed during frost heave. When the weather becomes warm, the ice melts to water while still in the corrugated pipe.

Numerical experiment verification

To verify that frost heave caused the crack of internal water in the corrugated pipe, this paper establishes a fine 3D model of the wall structure, as shown in Fig13. Through numerical evaluation, the stress distribution on the wall under water frost heave and the destruction form are analyzed. The result is compared with the actual detection outcome.

The finite element calculation model is adopted to determine a section of the 5-m long wall. The calculation model consists of the wall, pre-stress anchor cable and reinforcement, corrugated pipe, anchor and grout body, and pre-stress unit. A solid unit is used for modeling, which forms a total of 135,360 hexahedral elements.

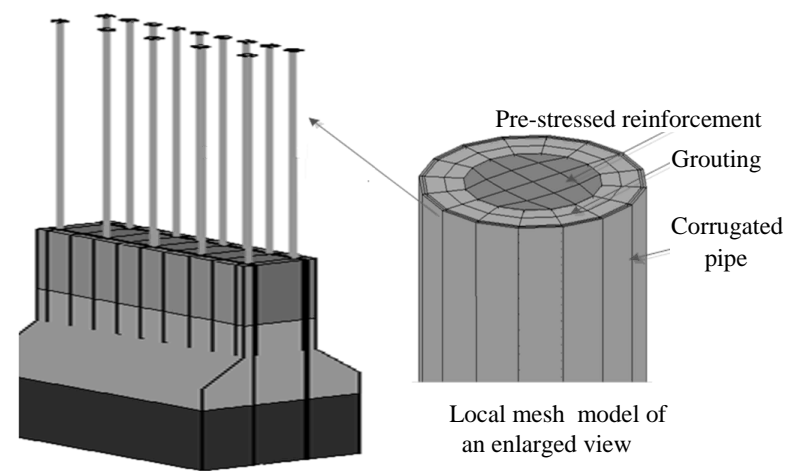

Fig. 13 Calculation model Local network model

For the numerical simulation, the linear elastic finite element method with prefabricated joints is adopted to calculate the occurrence, extension, and damage of the simulated cracks (Zhu 1998,Zhang 2011\&Zhang 2013). The calculation result is used to analyze the mechanism of the hollow crack.

Given the tensile strength and shearing strength of the crack unit, as seam stress failed to meet the seam strength criterion, the seam is in the bonding state. The force transmission mechanism of the seam is consistent with the seamless state. When the seam stress reaches the destruction intensity, the seam is pulled or cut. The strength criteria of the unit of the seam are as follows (Zhang 2011): 
(1) Tension fracture, $\sigma_{\mathrm{n}}>\sigma_{\mathrm{c}}$

(2) Shear failure

Compression shear, $\tau>c+\sigma_{\mathrm{n}} \mathrm{tg} \phi$

Shear, $\tau>c$

(3) Compression slip, $\tau>c^{\prime}+\sigma_{\mathrm{n}} \mathrm{t} g \phi^{\prime}$

where $^{\sigma_{\mathrm{n}}}$ refers to the normal tension stress of the seam; $\tau$ denotes the tangential shear stress;

$\sigma_{\mathrm{c}}$ indicates the tensile strength of the seam; c refers to the cohesion of the seam; $\phi$ denotes the friction angle of the seam surface; $C^{\prime}$ and $\phi^{\prime}$ indicate the remaining cohesion and friction angle, respectively.

Numerical simulation analysis result

Fig. 14 shows the full simulation process of elastic mechanics with prefabricated joints from initiation, expansion, and penetration. The simulation result indicates that the condition of the multi-bellow heaving when frost heave reaches $0.06 \%$, cracks on both sides start to appear (Fig. 14a); when frost heave reaches $0.28 \%$, cracks on both sides start to penetrate the concrete (Fig. 14b), and the crack in the middle starts to expand; when frost heave reaches $0.4 \%$, cracks on both sides and in the middle start to penetrate (Fig. 14c), and the internal cracks are connected to form a large area of hollow cracks.

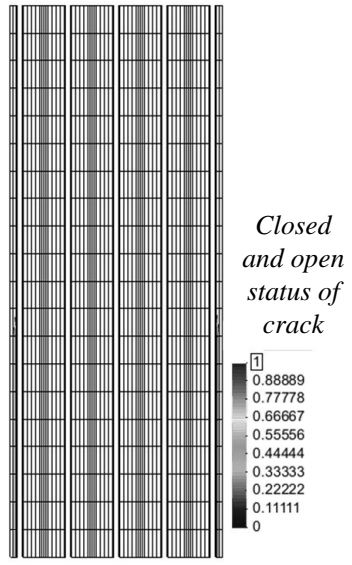

(a) Initiation

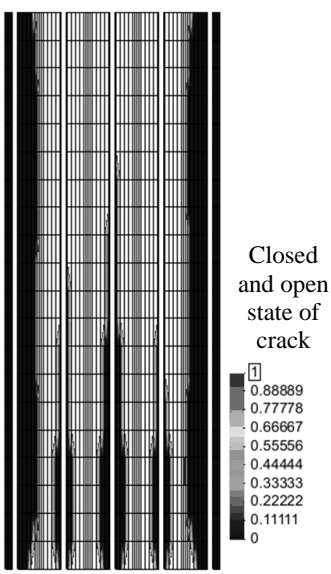

(b) Expansion 


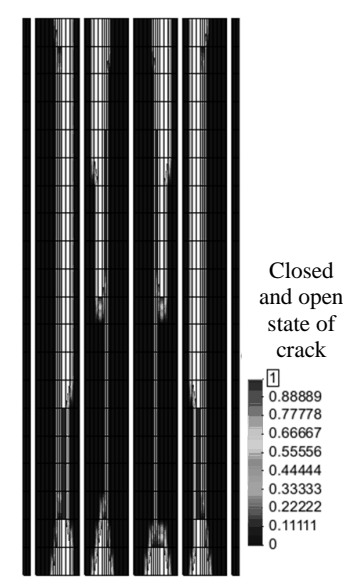

(c) Penetration

Fig. 14 Process of initiation, expansion, and penetration of longitudinal cracks on vertical wall when multi-bellow heaving occurs

The result of the multi-bellow heaving analysis is based on the assumption that many corrugated pipes are simulated at the same time and in the same process of frost heave. This situation is the most dangerous work condition. In the actual condition, the frost heave of each corrugated pipe is different. The simulation result reflects the difference from the shape of the hollow seam detected in the site. This outcome is caused by different assumed conditions. However, the frost heaving destruction of water in the corrugated pipe may be assumed to be the main cause of the internal cracks on the wall.

\section{Conclusions}

This paper introduces the detection principle of the impact imaging method, data acquisition, and data analysis method, as well as presents the detection results, of the internal crack of large-scale two-direction pre-stressed concrete walls of a certain water diversion project. The comprehensive analysis and 3D linear and nonlinear finite element numerical simulation result of the method are used to analyze the cause of cracks. According to the detection results and the analysis of the cause of cracks, the following conclusions are obtained:

(1) The impact imaging method is fast, highly efficient, and simple to use in detecting internal cracks, non-dense conditions, and other defects in pre-stressed concrete structures; thus, this method provides clear results.

(2) The impact imaging method can overcome the effects of multi-layer reinforcement net in the concrete and internal pre-stressed reinforcement in corrugated pipes to rapidly detect the internal defects of concrete.

(3) The core-taking verification findings indicate that this method is highly accurate, has good resolution, and provides reliable detection results.

(4) The disadvantage of this method is that numerical simulation and fewer core samples are needed to calibrate the result, i.e., to formulate a criterion that uses the impact response intensity to examine the internal defects.

(5) Analyzing the cause of cracks demonstrates that corrugated pipe grouting is not dense. In winter, the frost heave of water that accumulates in the corrugated pipe is the main cause of the internal cracks of walls.

This defect on the wall, which was attributed to low temperature, was identified and detected early. 
However, even if the water that has accumulated in the corrugated pipe does not turn to frost, the pre-stressed reinforcement rusts rapidly, thereby reducing the lifetime of the pre-stressed reinforcement. Thus, management and construction units should strengthen the control of grouting quality to ensure complete grouting of corrugated pipes and prompt closure of grouting holes. If necessary, secondary grouting must be included to prevent water accumulation in corrugated pipes.

\section{Acknowledgments}

This work was financially supported by the Governmental Public Industry Research Special Funds for Projects of Ministry of Water Resources of PRC (201201050), National Natural Science Foundation of China (51578544), and the Special Research Funds of IWHR.

\section{References}

[1] People's Republic of China Water Industry Standards(CWIS), 2005. Code for Engineering Geophysical Exploration of Water Resources and Hydropower(SL326-2005). China Water Conservancy and Hydropower Press, Beijing(in Chinese).

[2] Che Ai-lan, Huang Xin-chun, Guo Qiang et al, 2011. Evaluation of mud-jack method filling effect of immersed tube tunnel using surface wave survey method . Journal of Shanghai Jiaotong University, 45(5):648-652(in Chinese).

[3] Wan Li, Han Xiao-jian, Wang Bo et al, 2012. Experiment study on the impact-echo test for the flaw detection of concrete structure. Concrete, 2(268):8-10(in Chinese).

[4] Deng Zhong-jun, Yao Cheng-lin, Jia Yong-mei, et al, 2008. Application of ground penetrating radar to quality detection of hydraulic tunnel. Water Resources and Hydropower Engineering, 39(10):108-112(in Chinese).

[5] Liu Chao, Che Ai-lan, Feng Shao-kong,2013. Propagation characteristics of elastic wave in layered medium and applications of impact imaging method. Journal of Shanghai Jiaotong Univ. (Sci.), 18 (4):479-485.

[6] Huang Tao, Feng Shao-kong, Zhu Xin-min etc, 2015. Application of impact imaging method to detecting hollowing cracks wall of aqueduct.Water Resources and hydropower Engineering,46(1):60-64(in Chinese).

[7] Zhu Bofang,1998. Principle and application of finite element method. China Water Conservancy and Hydropower Press, Beijing(in Chinese).

[8] Zhang Guoxin, Liu Yi, Zheng Cuiying et al,2011. Simulation of influence of multi-defects on long-term working performance of high arch dam. SCIENCE China Technological Sciences, 54 (Suppl. 1):1-8.

[9] Zhang Guo-xin, 2013. Development and application of SAPTIS - a software of multi-field simulation and nonlinear analysis of complex structures (Part I). Water Resources and Hydropower Engineering, 44 (1):31-37(in Chinese). 